\title{
Culture meets surgery
}

\section{Hannes Haberl}

Published online: 5 November 2013

(C) Springer-Verlag Berlin Heidelberg 2013

Berlin treasures a very specific sentiment. In contrast to other old but traditionally wealthy German cities like Munich or Hamburg, Berlin never settled and never have the city's claims been staked. Although its importance ever increased, Berlin looks back to a rather checkered history of rise and fall, of unification, separation, and reunification. A flourishing trading city in the Middle Ages after merging the market towns Berlin and Coelln, it has experienced a first division and loss of independence in 1442. After reunification in 1470, Berlin until these days lived through an exhausting succession of political and economic crises causing repeated periods of substantial impoverishment and suppression, answered by organized resistance, civil unrest, and uprisings.

A basic flavor of depression and desperation remained alive at all times and may very well be experienced today in the somewhat harsh and gruffy sense of humor of native Berliners, which at most encourages to take eventual blows of faith relaxed and with composure. Somewhat unaffected by adverse events and as if shrugging off the misery, Berlin continuously developed. It became an electoral residence, royal city, and imperial capital. Under Frederick the Great Berlin was the intellectual and cultural center of enlightenment. In 1909, the first German motor airport opened. In the golden 20s, personalities like the architect Walter Gropius; the physicist Albert Einstein; the painter George Grosz; writers like Arnold Zweig, Berthold Brecht, and Kurt Tucholsky, and actors and directors like Marlene Dietrich, Friedrich Wilhelm Murnau, and Fritz Lang turned Berlin into the cultural capital of Europe. The nightlife of that period found its most famous description in the film Cabaret. Four million inhabitants celebrated the opening of the first

Culture meets Surgery was the title of the opening conference of the transdisciplinary research project, SchädelBasisWissen, directed by Sigrid Weigel and Hannes Haberl.

H. Haberl $(\bowtie)$

Division of Pediatric Neurosurgery, Charité-Universitätsmedizin, Berlin, Germany

e-mail: Hannes.Haberl@charite.de
Autobahn in 1921. In 1924, the airport Tempelhof was opened, and the first public television was introduced in 1935.

All this sounds very much like a success story if there had not happened the war of 30 years, halving the population, the occupation by Napoleon causing a sinister period of regression, followed by the first world war, bringing hunger and poverty, the Weimar Republic with harsh internal political hassles, followed by a galloping inflation and - after recovery by help of the allies - the word economic crisis, preparing the ground for national socialism and the second world war. Mutating into the capital of national socialism, Berlin experienced the horror of anti-Semitism, hardly suppressed during the Olympic Games of 1936 . The city finally was destructed for the greater part between June 1940 and February 1945 by the most numerous allied bombing raids ever flown over a German City during the second world war.

After a long period of vegetative survival, broken into pieces, and on the drip feed of the world powers, Berlin recovered slowly and only after the reunification in 1989 developed again into the current vibrant capital. This time, the traces of the past remained visible. The grounds of the former Berlin Wall, an only very tentatively built on fallow land, cut through the very city center, marking a virtually and very symbolic "broken heart". Thus, the city center from the palace of tears down to Checkpoint Charly breathes a strange mix of bitter and sweet memories, loading the place with sentimentality.

Alive but deeply marked by the fundamental crises of the past, Berlin continues its never ending journey towards a very distant and radiant goal. Berlin keeps on walking or rather strolling, admired, and accursed for this eternal status of a tramp - poor but confident. Particularly in summer, drowsily drifting along in the sun, this very emotional city seems to just dream away, aimlessly self-absorbed in its narcissism.

It is this specific mind setting which attracts - on the axis from Paris to Moscow - the shady world of glamour, illusion, gamble, fast wealth, and mass parties for any reason - be it the soccer world championship or the one mile high heels catwalk race-but as well creative potential, arts, and multiple scientific disciplines. Against this sparkling background, 
uncommon encounters on any level seem just more natural, and that is why Berlin probably was the most obvious host for the recent encounter of culture and surgery, two contrasting worlds, two different universes, yet sitting together on a cold winter weekend and listening with ever increasing fascination to each other's reports from alien worlds.

Invited by Sigrid Weigel (Head of the Berlin Center of Literary and Cultural Studies) and Hannes Haberl (Head of the Pediatric Neurosurgical Services/Charité/Berlin), an artist (Matt Jones/Story Artist/Pixar Studios/San Francisco), an artist in life sciences (Richard Neave/Forensic and Archeological Facial Reconstruction/Manchester), a cranio-maxillo-facial surgeon (Hans-Florian Zeilhofer/University Hospital of Basel), a computer professional (Stefan Zachow/Department of Visualization and Data Analysis, Numerical Analysis and Modelling/Zuse Institute/Berlin), a psychologist (Nichola Rumsey/Center for Appearance Research/University of the West of England/Bristol), a physician and historian of science (Michael Hagner/Science Studies/Swizz Federal Institute of Technology/Zürich), an expert in disabilities studies (Rosemarie Garland-Thompson/Women's Studies/Feminist Theory/ Disability Studies/Emory University/Atlanta), two art historians (Jeannette Kohl/History of Art/University of California/ Riverside, Martin Kemp/Scientific Models of Nature/Theory and Practise of Art/Art and Science/Art and Writings of Leonardo da Vinci/University of Oxford/Oxford), a medical historian (Thomas Schnalke/History of Medicine/Medical Museology/Charité/Berlin), an anatomist (Andreas Winkelmann/Human Remains Project/Charité/Berlin), three cultural studies experts (Sigrid Weigel/European Literature and Culture/Cultural History of Knowledge/Image Theory/Center for Literary and Cultural Research/Berlin, Uta Kornmeier/ European History of Exhibitions and Museums/Center for Literary and Cultural Research/Berlin, Simon Strick/History of Pain, Race, Gender and Sexuality in the History of Science/ Visual Culture/Center for Literary and Cultural Research, Berlin) and - last but not least-four pediatric neurosurgeons (James T. Goodrich/Montefiore Children's Hospital/Albert Einstein College of Medicine/New York, Concezio di Rocco/ Catholic University and Medical School/Rome, Mark R. Proctor/Children's Hospital/Harvard Medical School/Boston, Hannes Haberl/Charité/Berlin) discussed "Images, Models, and Interpretation of the Human Skull."

\section{Why this?}

In the view of ever developing technological options, I feel a growing unease about the stagnation of surgical techniques pioneered in the 1960s by Paul Tessier and performed all over the world by more less the same rules for almost 60 years now. At the same time, there is a rising awareness of society concerning esthetic questions, which seems to be taken up only very hesitantly by the protagonists of a predominantly esthetic procedure. This hesitation may have historic reasons.

After a history of repeated cultural overload of the meaning of head shapes most recently by anthropology and racial biology in the first half of the twentieth century, substantiating the practices of racial extermination executed by different colonial administrations and national socialists, science has entirely lost sight of the skull.

Also, by the upcoming neurosciences, the skull was barely regarded as more than a vessel for the brain. This indifference has preserved a white spot on the map of not only surgery but also science: There is very little anatomical material covering the dimensions and proportions of the growing skull in early childhood, and there is a certain reluctance of the concerned disciplines to deal with the medical or cultural implications of head shapes as terminology and visual presentation are burdened by history. Needless to add that there are also no established specific goals for reshaping skulls.

Despite this poor scientific support, synostosis surgery is exposed to a daily and substantial pressure to act, underlining the importance of developing the field and also tackle the following — in parts uncomfortable — issues which might need other than medical expertise.

\section{Reshaping is esthetic surgery}

Independent from still controversially discussed functional issues, cranial reshaping tries to achieve an esthetically convincing late result in every case. In the vast majority of single suture synostoses, the surgical indication is preponderantly based on esthetic concerns. For this large group, the circumstances of elective esthetic surgery change the framework for the communication between surgeon and parents in many ways.

Surgeons take much responsibility committing a "willful bodily injury" on a child. While other than esthetic conditions leave very little doubt in the public understanding that surgery is indicated if it is the most efficient way to improve the child's physical condition at the price of a manageable risk, the point of departure in esthetic surgery is slightly different. Particularly in single suture synostosis, the amount of anticipated social impairment represents the basis of a surgical indication. Thus, the indication is referring to a deviation from a cultural convention on normality. The pressure to act is derived from a very subjective and individual interpretation of the impact of this deviation on the very child.

In a first step, both the surgeon and the parents have to weigh the assumed impact-i.e., a future social impairment caused by a visible malformation - in relation to the surgical risk. Addressing aspects like a life with a visible malformation versus a life with a surgically corrected appearance, respectively identity is not only very inconvenient, it probably 
exceeds medical and also parental expertise. Furthermore, the detailed imagination of a severe handicap caused by surgery is probably unbearable. The consideration of the worst case, which can be the death of a child, definitely overstrains the emotional potential of both parties. I therefore assume that consent usually is and probably has to be achieved and signed without a full risk benefit assessment.

Other than in functionally indicated surgery, the relationship between surgeon and patient in esthetic surgery is not defined by the natural imbalance between the professional position of a medical expert disposing of a knowledge advantage versus the indigent position of a suffering and dependent patient. Instead, the surgeon's as well as the parents' opinion is based on nothing but implicit esthetic knowledge. Probably more common in plastic surgery, this creates an unfamiliar situation for a neurosurgeon who is used to communicate from a superior perspective arising from his potential to make the invisible visible; to look by means of radiation, ultrasound, or magnetic fields into the body and "see" the disease. The persisting claim of many surgeons to do x-rays, MRI, or CT scans also in single suture synostosis despite the lack of substantial additional information may not least be motivated to some extent by the defense of this superior position.

\section{Reshaping is "constructive" surgery with a fictitious goal setting}

In contrast to "reconstructive" surgery, following a matrix defined by pre-existing patient-derived data and trying to restore a pre-existing appearance, craniofacial surgery in children already born with a malformation per definition has to be a free creation. Reference can only be provided either independently from the individual patient's appearance by "normal" head shapes of other children or by arbitrary changes of the (pathologic) data set of the patient's appearance using virtual planning tools [1]. As in most surgical units, both types of reference are not provided, they have to be replaced by a fictitious model which is represented either in the personal imagination of the surgeon or in a surgical "standard", which defines the rules of appropriate surgical relocation of bone fragments by two-dimensional statistical measures according to an imagination provided by the authors of these rules. All of those currently used alternatives are either completely or partially based on fiction.

\section{Missing quantitative and qualitative goals result in a lack of follow-up criteria}

Without the reference of a defined surgical goal, a convention on follow-up criteria is not possible and has never been achieved. To this day, the evaluation of surgical results is commonly based on two-dimensional photographs or measures [2], offering a pre-post comparison which either cannot be validated or does not fully represent the threedimensional result. A reliable, quantifiable database of results is not available.

Based on the analysis of 79 publications on craniosynostosis surgery between 2003 and 2012, ${ }^{1}$ the description of the surgical goal of remodeling remains very vague and is focused on the repeatedly used terms "improvement of facial attractiveness," "softening the forceful look," achieving a "more pleasant expression," "improving appearance," achieving "symmetrical recontouring". Although these terms suggest an impact on quality of life, the use of corresponding scales is not customary.

\section{The development of surgical techniques is stagnating}

Common surgical standards offer a range of two-dimensional measures, based on not validated surgical results and leave the determination of the exact shape of invariably curved bone fragments, forming a three-dimensional space, to the surgeon's subjective "experience." There is of course a treasure of experience in the field, and the overall impression most surgeons would agree on is that current techniques of cranial reshaping in experienced hands do make a lasting difference, though this impression still is to be validated and refined to describe the true range of achieved results and to prove and exploit the full potential of new technologies. To this day we - for example - do not know to which extent the amount of frequently observed loss of correction follows an age-dependent algorithm or is driven by individual factors. Only recently, normal range data base-derived statistical models are entering the scene [3, 4], offering the opportunity to replace subjective, esthetic, or intuitive criteria by exact statistical information. Not only the provision of appropriate data but also their precise transformation into surgery is a task still to be performed and subject of one of our current research projects.

Probably the strongest motivation for improvement at all times is dissatisfaction. As long as the evaluation of results remains based on subjective criteria, it will be impossible to create evidence. I am very concerned that dissatisfaction regarding the results of craniofacial reshaping currently is successfully avoided by delaying evidence although the required technical tools are available.

As a consequence of the above mentioned conditions, I do see several major tasks for the future development of cranial reshaping.

$\overline{{ }^{1} \mathrm{CNS} / 54, \text { JNS}}$-pediatrics /19, Aesthetic Plast Surg/1, J Craniofac Surg/4 


\section{Clinical challenges}

Improvement of evaluation tools

Quantifiable evaluation criteria are inevitable. To describe the status quo, to define the potential of classical surgical strategies, and-most important-to realize and proof the potential of new surgical tools, three-dimensional scans or three-dimensional photographs providing information on local volume and shape changes should be used for a thorough and plausible documentation of surgical results. Agedependent growth patterns, monitoring for instance the ratio of loss of correction then can directly feedback to the used surgical technique, establishing a learning system. They can also be implemented into the choice algorithm of appropriate (standard) models for a future model-based surgery allowing for appropriate overcorrection if necessary.

The acceptance of technological improvements and novel procedures will increasingly depend on a simultaneous measurement of costs and effectiveness, particularly taking into account morbidity, mortality, and generic, specific, and utility QOL scales [5, 6]. From a cultural perspective, the objectivity of QOL scales is doubtful as surgical indication and evaluation of results is referring to the amount of assumed social impairment derived from a very subjective and individual interpretation of the impact of a deviation from normal on the very child.

\section{Improvement of surgical tools}

Navigated surgery based on the transfer of radiological data into a surgical procedure has become a common tool in neurosurgical theaters. Its purpose is obvious as long as the task is to reach an existing defined point in space within the body of the patient, which can be displayed on an appropriate radiological examination. However, the task in craniosynostosis surgery is to create a novel, non-existing "physiological" position for any referring point on the patients deformed skull which might as well be outside the current body limits and which therefore is not representable by radiological examinations.

Instead of radiological data, navigation would therefore need a different reference, which is defined by a fictitious physiological model other than the patient's authentic skull. This model can be - and currently frequently is - the result of a creative act of the (planning) surgeon based on his esthetic knowledge. In contrast, it could also be the result of a statistical analysis of already existing physiological head shapes of comparable children of the same age derived from a databank.

Our experiences with a developing data bank, allowing for the reproduction of customized compositions of individual skull reconstructions, are encouraging, and the first surgical results using these models are submitted for publication.
One major advantage of using data, which are already available in many radiological archives around the globe is the prevention of potentially harmful and stressing individual radiological diagnostics. The creation of a standardized set of shape models would also safe costs rendering individual diagnostic examinations as well as surgical planning unnecessary as the navigation data then virtually are already on the surgeon's table represented in a physical threedimensional model.

To transfer the information represented in the model into the very refractory bone fragments of the child, solid mechanical tools are required. A customized frame is under construction, and I am sure that many other solutions will be developed in the near future.

The fundamental challenge for future craniosynostosis surgery will be to avoid stagnation by autistic insistence on traditions. Extensive surgery in the youngest must be made accessible for already existing contemporary technological solutions.

\section{Transdisciplinary challenges}

Craniosynostosis surgery is as much of a cultural as a medical tool

The desire to improve the quality of treatment is shared by many colleagues. In 2010, "Craniosynostosis: Developing Parameters for Diagnosis, Treatment, and Management" [7], a North American multidisciplinary initiative of 52 attendees in 2010, representing a broad range of expertise including anesthesiology, craniofacial surgery, dentistry, genetics, hand surgery, neurosurgery, nursing, ophthalmology, oral and maxillofacial surgery, orthodontics, otolaryngology, pediatrics, psychology, public health, radiology, and speech-language pathology started to create parameters of care for individuals with craniosynostosis and thus improve medical care by providing common access to a defined standard of treatment.

However, esthetic surgery is as much of a cultural as a medical tool. Therefore, it is probably not perfectly accommodated in a hermetically sealed exclusively surgical environment. Our current transdisciplinary project looks at the hitherto less questioned cultural background of current medical practice. Addressing issues, which are difficult to be dealt with in a busy surgical daily routine, need a new platform for surgeons and cultural scientists. Our project is an attempt to involve nonmedical disciplines into the ongoing discussion on craniosynostosis surgery.

The skull is cult. Skull images remain to belong to the most reproduced pictures ever. Though, in research, after a period of overload of meaning, the significance of the skull has regressed to serving as a platform for brain function and facial expression. Since the rejection of the physiognomic idea, the skull receives only limited attention for instance in evolutionary biology with 


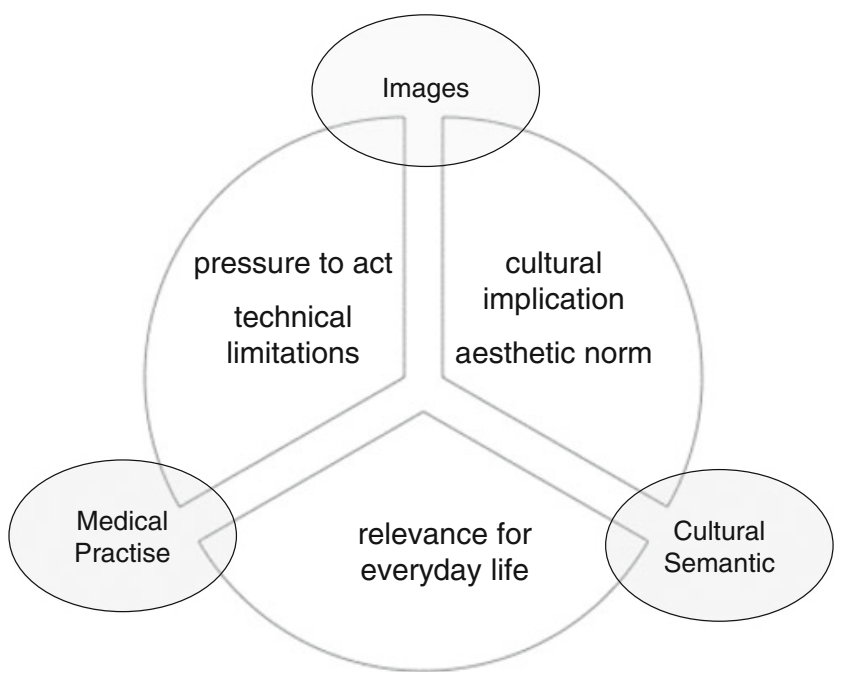

Fig. 1 Cultural implications of medical practice respect to the coevolution of skull brain and face. The obvious relevance of the skull shape for the perception of the individual seems to be of subordinate interest. This rather academic order of values changes when a patient and his treating physician have to deal with a skull malformation. Suddenly, the social impact of a deviation of the normal becomes the compelling argument for very invasive surgery. Associating the craniofacial shape with individual behavior seems to be an unavoidable phenomenon of interaction, and great effort is taken to avoid what is considered to be an undesirable attribution. Without returning to the paradigms of the nineteenth century, the origin of this involuntary reaction still is to be analyzed, be it an archaic species-related reaction, the aftermaths of outdated physiognomic knowledge, or a product of the international deluge of omnipresent pictures establishing increasingly uniform esthetic standards.

The discussion of surgical indications, goals, and results reveals a baseline of common sense regarding contemporary esthetic concepts. The vocabulary of this discussion is
Fig. 2 Culture meets surgery: see you at the Brandenburg Gate....!

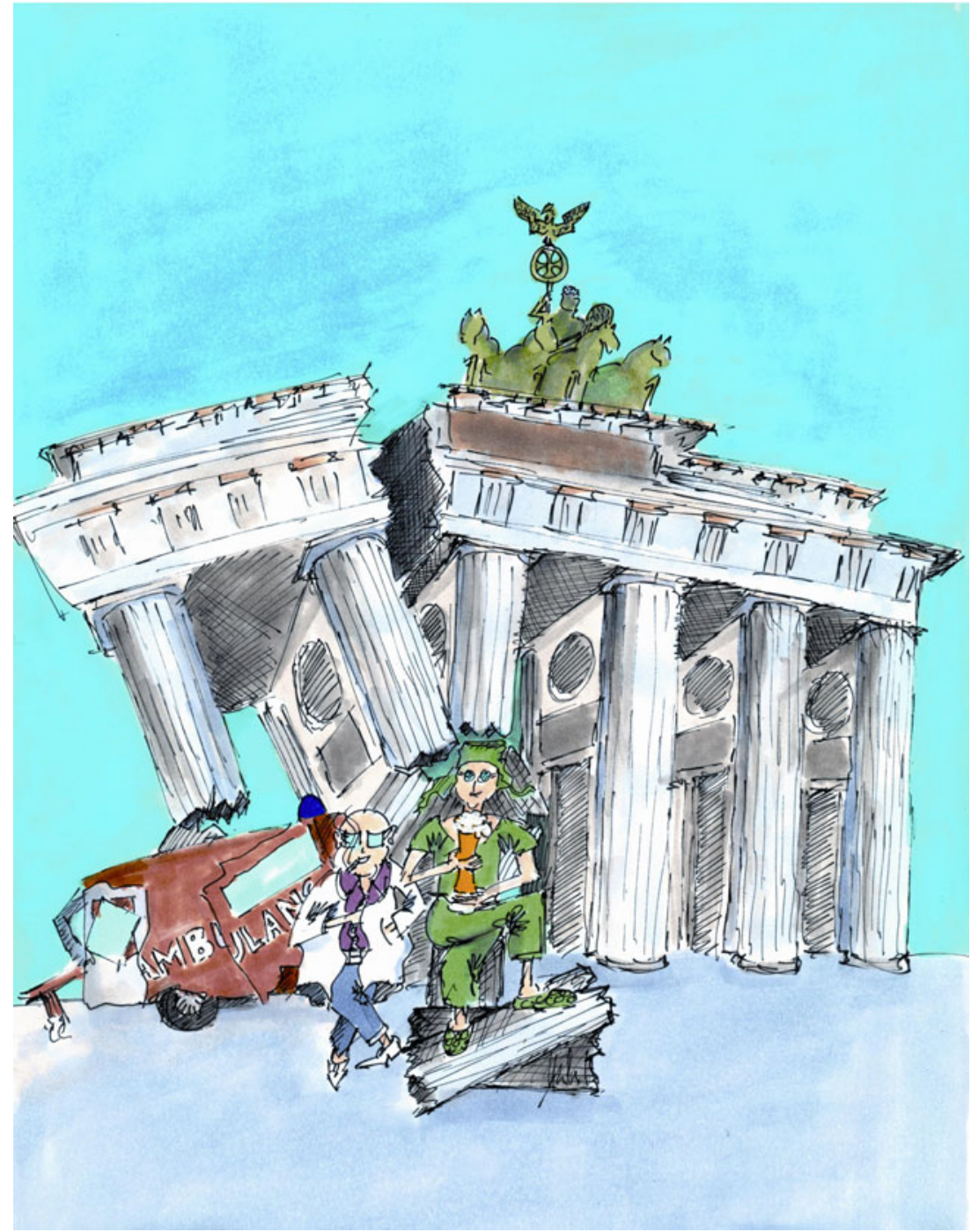


composed of medical, historical, and popular texts, images in art, medicine, cinema and on the street adding up to an unreflected implicit esthetic knowledge. The presence of a malformation and the option of a surgical intervention connect all these components with current medical practice to a dynamic system of interaction (Fig. 1), which is the object of inquiry of our current project.

The social significance of a surgical procedure cannot be assessed by (only) the surgeon

Sigrid Weigel once stated, that "....accelerated by increasing sub-specialization, cultural context, historical conditions, profit, limits, and side effects of (medical) innovations cannot be overseen anymore by only the involved scientists, cultural expertise is required..." This is particularly true for surgery, which is largely driven by the assumed social impairment of a deviation from the normal.

The goals of this study will be to analyze the cultural implications of medical practice. Narrative strategies, terms, criteria, metaphors, and imagination will be evaluated regarding their origin and their current meaning in medical literature as well as in physician-patient communication.

The patient perspective as exposed during the communication with the surgeon as well as during separate interviews will be further analyzed. Anatomical and proportional studies in art and medicine and their use in current publications, software applications, information material, and popular communication media will be studied. Another aspect will be the interaction of medical and artistic "body knowledge" as used by a surgeon compared to a sculptor.

This multifocal approach is expected to provide various results:

1. Provision of context knowledge

Context knowledge will be allocated by publications for patients, scientists, and the general public in order to reinforce the decision-making competence of surgeons and concerned families.
2. Provision of information material

Information material for surgeons will be provided to increase the awareness and to support the critical reflection of implicit esthetic knowledge and norms.

3. Update of the interaction of medicine, art, and cultural semantics

Last but not least, the evaluation of the cultural implications of craniosynostosis surgery will serve as pars pro toto, as the interaction of medicine, art, and cultural semantics in many medical fields may profit from an update. Please feel invited to participate and share your thoughts with cultural scientists and surgeons in an upcoming series of shortly announced winter workshops 2013 which will take place...guess where.....(Fig. 2)

\section{References}

1. Diluna ML, Steinbacher DM (2012) Simulated fronto-orbital advancement achieves reproducible results in metopic synostosis. J Craniofac Surg 23(3):e231-e234

2. Szpalski C, Weichman K, Sagebin F, Warren SM (2011) Need for standard outcome reporting systems in craniosynostosis. Neurosurg Focus 31(2):E1. doi:10.3171/2011.6.FOCUS1192. Review

3. Haberl H, Hell B, Zöckler MJ, Zachow S, Lamecker H, Sarrafzadeh A, Riecke B, Lanksch WR, Deufelhard P, Bier J, Brock M (2004) Technical aspects and results of surgery for craniosynostosis. Zentralbl Neurochir 65(2):65-74, May

4. Saber NR, Phillips J, Looi T, Usmani Z, Burge J, Drake J, Kim PC (2012) Generation of normative pediatric skull models for use in cranial vault remodeling procedures. Childs Nerv Syst 28(3):405410, Epub 2011 Nov 17

5. Lee HQ, Hutson JM, Wray AC, Lo PA, Chong DK, Holmes AD, Greensmith AL (2012) Analysis of morbidity and mortality in surgical management of craniosynostosis. J Craniofac Surg 23(5):1256-1261

6. Thoma A, Ignacy TA (2012) Jan Health services research: impact of quality of life instruments on craniofacial surgery. J Craniofac Surg 23(1):283-287

7. McCarthy JG, Warren SM, Bernstein J, Burnett W, Cunningham ML, Edmond JC, Figueroa AA, Kapp-Simon KA, Labow BI, PetersonFalzone SJ, Proctor MR, Rubin MS, Sze RW, Yemen TA, Group CW (2012) Parameters of care for craniosynostosis. Cleft Palate Craniofac J 49(Suppl):1S-24S 